\title{
Supplementary Info for "Multi-Scale Sea Ice Kinematics Modeling with a Grid Hierarchy in Community Earth System Model (version 1.2.1)"
}

This supplementary file includes 3 figures that accompany the manuscript "MultiScale Sea Ice Kinematics Modeling with a Grid Hierarchy in Community Earth System Model (version 1.2.1)”.
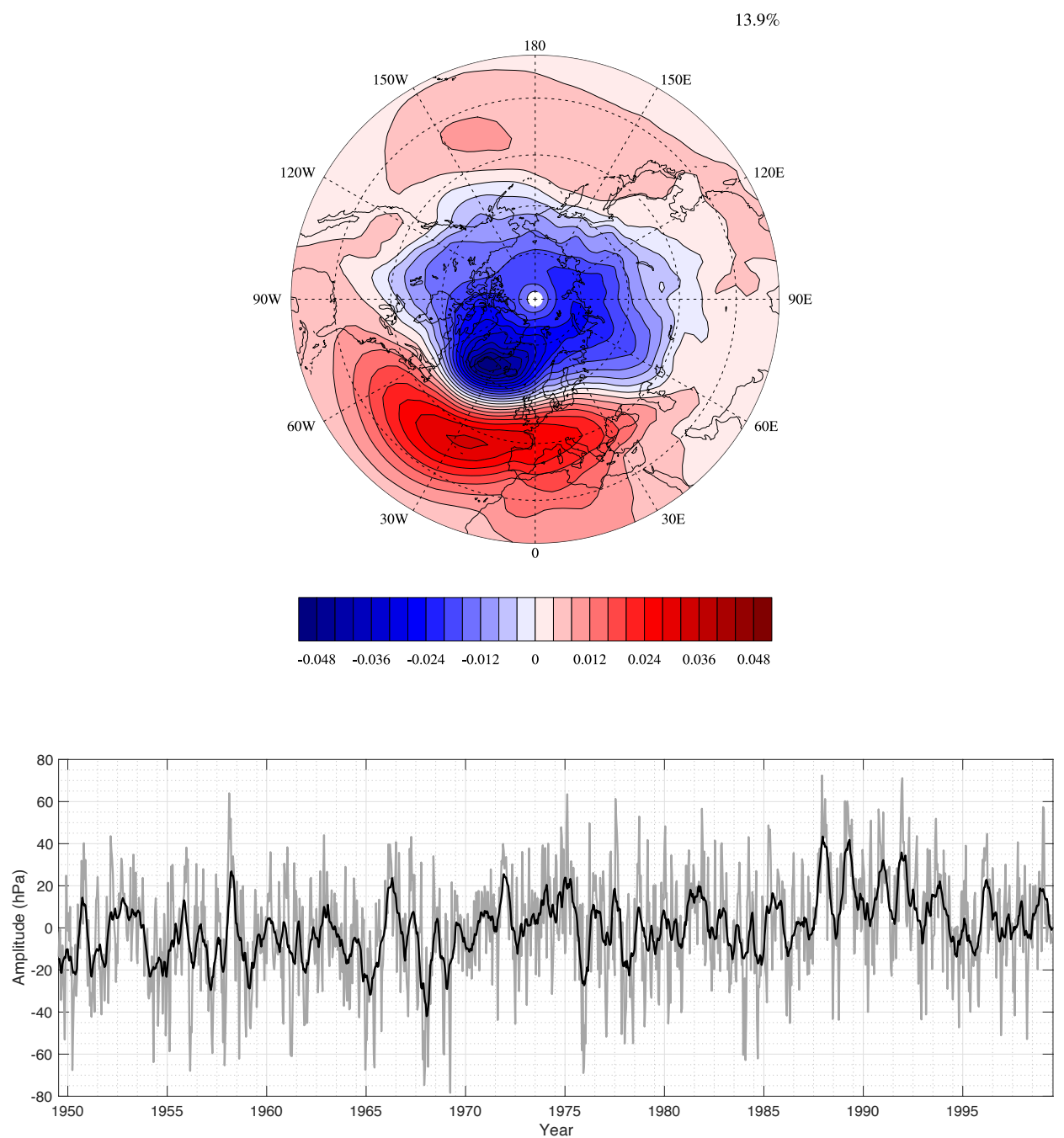

Fig. S1 Arctic Oscillation mode and principle component (PC) from 50-year wintertime SLP fields of NCEP reanalysis. The spatial pattern is normalized and the PC carries the same unit of the SLP anomaly $(\mathrm{hPa})$. 

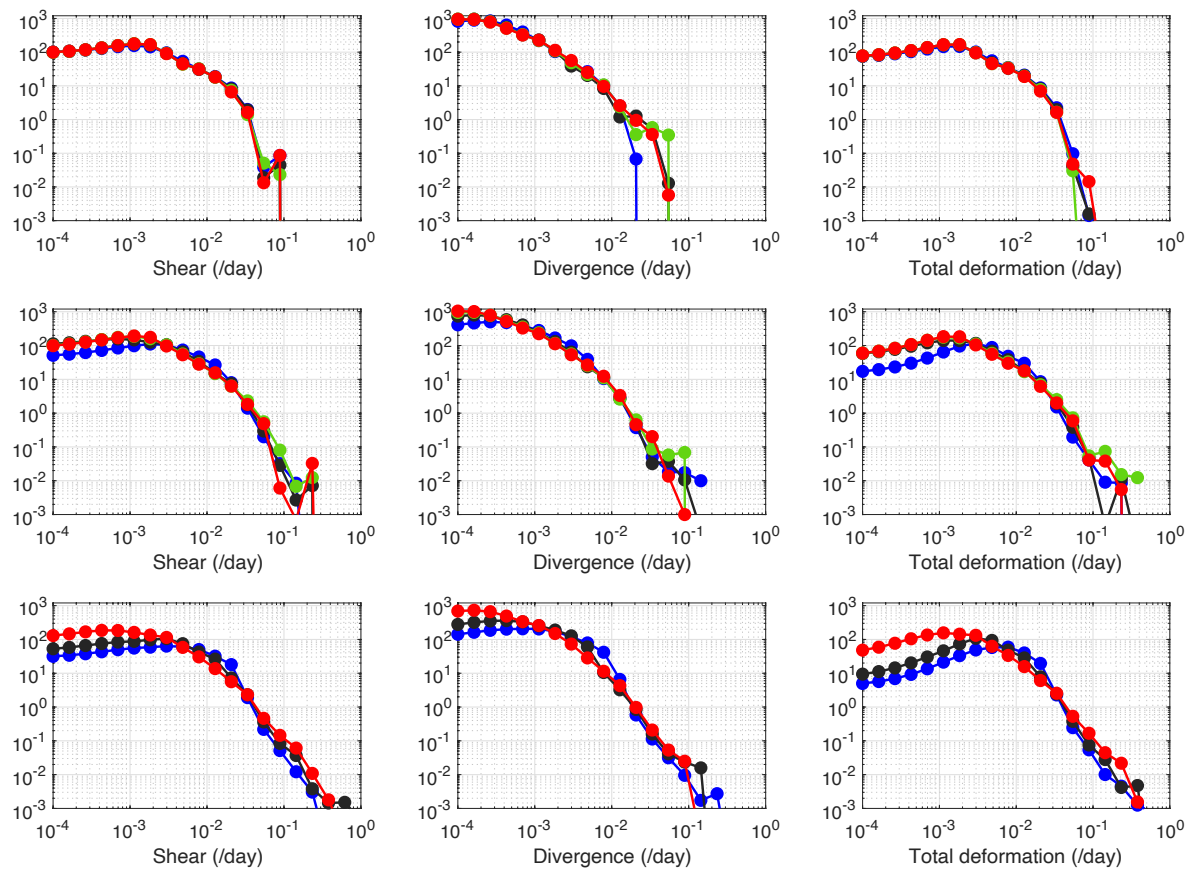

Fig. S2 Distribution of total deformation rate on Dec. 20th. First row, second row and third row represent the model output from TS045, TS015 and TS005, respectively. Shearing rate, divergence rate, and total deformation rate in the left, central and right column, respectively. The lines are color-coded as follows: blue for NDTE $=120$, black for $\mathrm{NDTE}=240$, green for $\mathrm{NDTE}=480$, and red for $\mathrm{NDTE}=960$. 

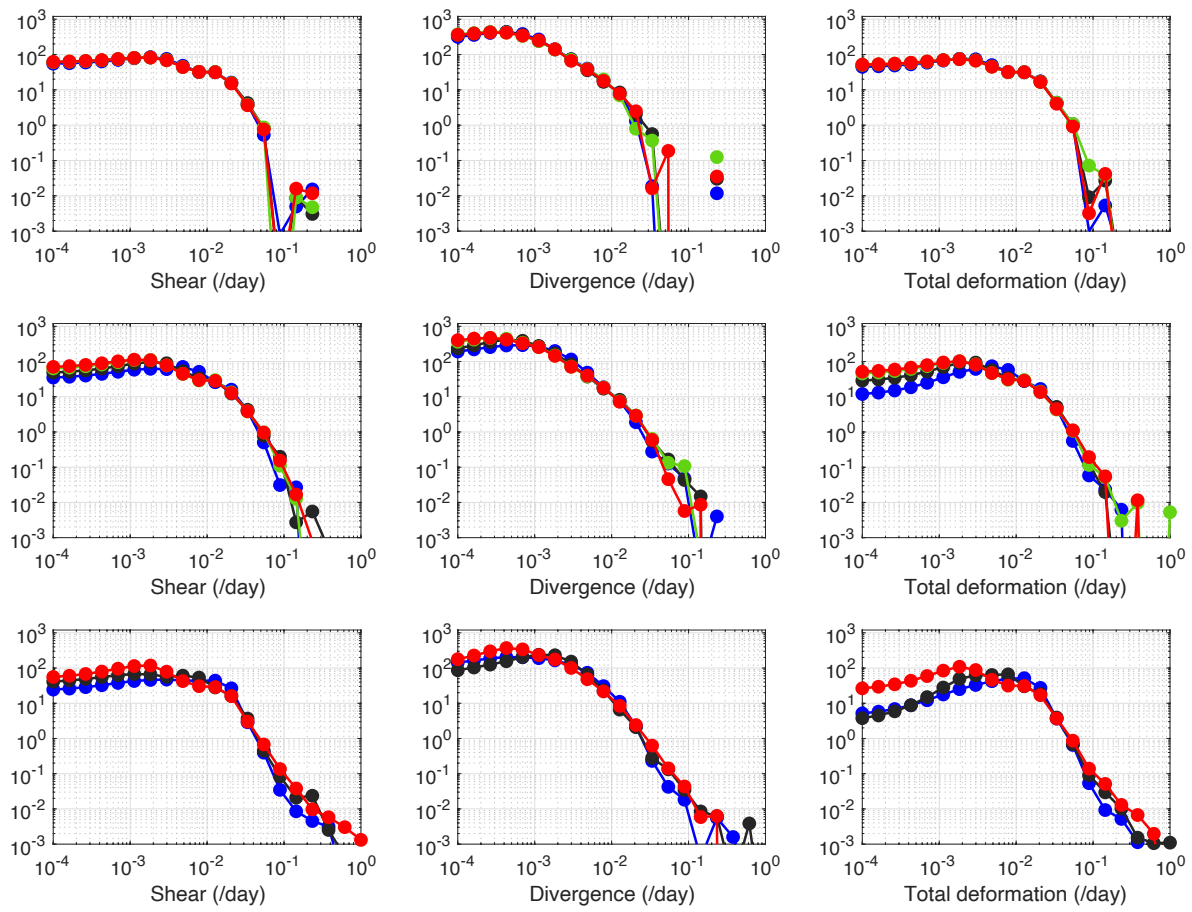

Fig. S3 Same as Fig. S2, but for Feb. 6th. 